\title{
Efecto de la adición de antioxidantes en el diluyente de semen de macho cabrío antes de congelar y después de descongelar
}

\author{
Membrillo-Ortega, A. ; Córdova-Izquierdo, A.; Hicks-Gómez, J.J.2; \\ Valencia-Méndez, J.J. ${ }^{3}$; Castillo-Juárez, H. ${ }^{1}$ \\ ${ }^{1}$ Universidad Autónoma Metropolitana Unidad Xochimilco. Departamento de Producción Agrícola y Animal. \\ Calzada del Hueso 1100 Colonia Villa Quietud, 04960, acordova@correo.xoc.uam.mx, México D.F. \\ ${ }^{2}$ Secretaria de Salud, Instituto Nacional de Enfermedades Respiratorias, México. \\ ${ }^{3}$ Facultad de Medicina Veterinaria y Zootecnia, Universidad Nacional Autónoma de México.
}

\begin{abstract}
Resumen
Membrillo-Ortega, A.; Córdova-Izquierdo, A.; Hicks-Gómez, J.J.; Valencia-Méndez, J.J.; Castillo-Juárez, H.: Efecto de la adición de antioxidantes en el diluyente de semen de macho cabrío antes de congelar y después de descongelar. Rev. vet. 22: 2, 85-90, 2011. $\mathrm{El}$ objetivo de este trabajo fue evaluar el efecto antioxidante de las vitaminas $\mathrm{C}$ y E en el semen de macho cabrío antes y después de descongelar y su efecto sobre la condensación de la cromatina espermática y el grado de oxidación de los espermatozoides. Se utilizaron cuatro machos cabríos de la raza Alpino Francesa y se analizaron ocho eyaculados de cada semental. Cada eyaculado fue dividido en cuatro grupos: control, con vitamina $\mathrm{C}$, con vitamina $\mathrm{E}$ y combinación de $\mathrm{C}+\mathrm{E}$. La determinación de la condensación de la cromatina espermática se realizó mediante citometría de flujo, utilizando el fluorocromo yoduro de propidio. Los resultados no mostraron diferencia estadísticamente significativa en el semen antes de congelar y después de descongelar $(\mathrm{p}>0,05)$, lo cual indica que con la adición de antioxidantes, la condensación de la cromatina espermática no registró variaciones. Para valorar el grado de oxidación de los espermatozoides se utilizó la reacción del ácido tiobarbitúrico, la cual puede detectar peroxidación de los lípidos de la membrana espermática. Se encontró que antes de congelar el semen no hubo diferencia estadísticamente significativa entre los grupos control, con vitamina $\mathrm{C}$, con vitamina $\mathrm{E}$ y combinación de $\mathrm{C}+\mathrm{E}(\mathrm{p}>0,05)$. Después de descongelar las muestras hubo diferencia significativa entre el grupo control y la mezcla de vitaminas $\mathrm{C}+\mathrm{E}$; se estimó que la combinación de antioxidantes redujo el estrés oxidativo $(\mathrm{p}<$ $0,05)$, siendo menor la cantidad de nanomoles de malondialdehido por miligramo de proteína. Se concluye que la adición de antioxidantes al diluyente, puede reducir el estrés oxidativo causado por la congelación y descongelación de los espermatozoides; sin embargo, no hubo efecto alguno sobre la condensación de la cromatina espermática.
\end{abstract}

Palabras clave: macho cabrío, semen, criopreservación, radicales libres, antioxidantes, peroxidación lipídica.

\begin{abstract}
Membrillo-Ortega, A.; Córdova-Izquierdo, A.; Hicks-Gómez, J.J.; Valencia-Méndez, J.J.; Castillo-Juárez, H.: Effect of the addition of antioxidants in the extender of semen of male goat before freezing and after thawing. Rev. vet. 22: 2, 85-90, 2011. The aim of this study was to evaluate the effect of vitamins $\mathrm{C}$ and $\mathrm{E}$ as antioxidants in goat semen before freezing and after thawing and their effect on sperm chromatin condensation and degree of oxidation of sperm. Four French Alpine males were used. Eight semen samples of each male were analyzed. Each ejaculate was divided into four groups: control, vitamin C, vitamin E and combination of $\mathrm{C}+\mathrm{E}$. The determination of the condensation of sperm chromatin was performed by flow cytometry using fluorochrome propidium iodide. Results showed no statistically significant difference in semen before and after freezing $(\mathrm{p}>0.05)$, indicating that the addition of antioxidants did not cause variations in the condensation of sperm chromatin. To assess the degree of oxidation of sperm before and after freezing, thiobarbituric acid reaction was used, which can detect lipid peroxidation of sperm membrane. Before freezing semen, no statistically significant difference was found between the analyzed groups: control, vitamin $\mathrm{C}$, vitamin $\mathrm{E}$ and combination of $\mathrm{C}+\mathrm{E}$ ( $\mathrm{p}>0.05)$. After thawing the samples of the same groups, statistically significant difference between the control and the mixture of vitamins $\mathrm{C}+\mathrm{E}$ was found $(\mathrm{p}<0.05)$, perhaps because the mixture of antioxidants reduced
\end{abstract}


oxidative stress; the quantity of nanomoles of malondialdehyde for milligram of protein was smaller. We conclude that the addition of antioxidants to the diluent can reduce the oxidative stress caused by freezing and thawing of sperm cells; on the contrary, there was no effect on chromatin condensation.

Key words: male goat, semen, cryopreservation, free radicals, antioxidants, lipid peroxidation.

\section{INTRODUCCIÓN}

El éxito de la congelación del semen depende de numerosos factores peculiares de cada especie y deben ser optimizados de acuerdo al tipo de semen que se va a preservar. Para la fecundación del ovocito de la vaca, se necesitan menos millones de espermatozoides que en la cerda, la cual requiere cantidades sensiblemente mayores. Esta diferencia cuantitativa entre las especies constituye una desventaja cuando se utiliza semen criopreservado, debido a la menor supervivencia espermática inducida por el proceso de congelación ${ }^{18}$.

El proceso de congelación de semen da como resultado un aumento en el número de células apoptóticas en comparación con el semen fresco ${ }^{1}$. Durante la criopreservación del semen, el estrés provoca daños en la integridad de la membrana con la consecuente pérdida de la motilidad y viabilidad, por lo cual la fertilidad del semen congelado es más baja comparada con el semen fresco, debido a la pérdida de capacidad de fecundación ${ }^{20}$. Dentro de los principales factores perjudiciales durante la criopreservación figuran los ocasionados por los radicales libres que se forman en este proceso ${ }^{8}$ siendo los responsables del daño oxidativo ${ }^{3}$.

El daño a bajas temperaturas ocurre en la membrana plasmática, en la membrana acrosomal, en la mitocondria y en la vaina del axonema ${ }^{24}$ y aunque fisiológicamente se forman radicales libres durante la respiración mitocondrial, es importante tener en cuenta que las anomalías en la mitocondria pueden contribuir a la producción excesiva de los radicales libres ${ }^{30}$. La generación de especies reactivas del oxígeno (ROS) por espermatozoides dañados tiene un impacto negativo sobre las células viables restantes, ya que representa un daño acumulativo para los espermatozoides que son almacenados ${ }^{3}$.

Entre las ROS destacan fundamentalmente el anión superóxido $\left(\mathrm{O}_{2}{ }^{-}\right)$, el hidroxilo $(\cdot \mathrm{OH})$ y el peróxido de hidrógeno $\left(\mathrm{H}_{2} \mathrm{O}_{2}\right)^{27}$. Este último, a pesar de ser un radical libre, es la molécula que más se ha involucrado en el daño de los espermatozoides de equino. El peróxido de hidrógeno es una molécula muy reactiva y puede ser precursora de radicales hidroxilo en presencia de metales de transición ${ }^{17}$. La reacción inicial de la oxidación de los ácidos grasos consiste en una lipoperoxidación y es generada por las ROS que inducen una reacción en cadena, provocando un rompimiento de dobles enlaces en los lípidos de las membranas ${ }^{28}$.

En espermatozoides humanos las ROS dañan los fosfolípidos de la membrana y están implicados en la infertilidad masculina ${ }^{23}$. Los cambios en el contenido de los lípidos pueden ser explicados por la lisis de los fosfolípidos de la membrana seguidos por cambios del metabolismo celular interno o por una compleja combinación de lisis, metabolismo y peroxidación. La peroxidación de los ácidos grasos puede ser analizada por medio de la cuantificación de malondialdehido, un indicador de lipoperoxidación de los lípidos de la membrana celular ${ }^{22}$.

Las ROS cumplen una importante función en la fisiología espermática normal, ya que pueden activar al espermatozoide en la fecundación ${ }^{17}$; un desequilibrio entre su producción y degradación causa efectos adversos sobre el espermatozoide ${ }^{5}$, provocando un estrés oxidativo que se ha definido como un desequilibrio entre los agentes oxidantes y los mecanismos antioxidantes. Estos últimos involucran sistemas enzimáticos y moléculas orgánicas diversas entre las que se incluyen las vitaminas $\mathrm{E}$ y $\mathrm{C}$, que cumplen su función antioxidante al disminuir el porcentaje de peroxidación lipídica ${ }^{2}$.

La vitamina $\mathrm{C}$ al donar electrones al radical tocoperoxil de la vitamina $\mathrm{E}$ oxidada recicla la función antioxidante del $\alpha$-tocoferol, ayudando a proteger a la membrana lipídica de la peroxidación, por lo cual la vitamina E sólo puede ser efectiva en combinación con la vitamina $\mathrm{C}^{12}$. Además se ha demostrado que con el proceso de congelación y descongelación, la cromatina espermática sufre importantes cambios que pueden influir en una muerte embrionaria temprana ${ }^{14}$.

El objetivo de este trabajo fue evaluar el efecto de las vitaminas $\mathrm{C}$ y $\mathrm{E}$ como antioxidantes en el semen de macho cabrío antes de congelar y después de descongelar, así como su efecto sobre la condensación de la cromatina espermática y grado de oxidación de los espermatozoides.

\section{MATERIAL Y MÉTODOS}

Animales. Se utilizaron 4 machos cabríos de la raza Alpino Francesa, de probada fertilidad, entre 2 y 4 años de edad, mantenidos en corrales individuales en las instalaciones del Centro de Enseñanza Práctica e Investigación en Producción y Salud Animal (CEPIPSA) de la Facultad de Medicina Veterinaria y Zootecnia de la Universidad Nacional Autónoma de México, alimentados con una ración de ensilado de maíz y alimento balanceado comercial.

Colección de semen. Se obtuvieron 8 eyaculados por semental; la colección del semen se realizó por medio de una vagina artificial a una temperatura inter- 
na de $42^{\circ} \mathrm{C}$. Una vez colectado el semen se colocó en bañomaría a $37^{\circ} \mathrm{C}$ para valorar parámetros como volumen, movilidad y concentración.

Valoración seminal. El volumen del eyaculado fue determinado en un tubo colector graduado; la movilidad en masa y la movilidad individual fueron determinadas con una microgota $20 \mu \mathrm{l}$ de semen colocada en un portaobjetos a $37^{\circ} \mathrm{C}$ y observada en microscopio de contraste de fase con una magnificación de 100x. La movilidad individual fue obtenida por observación de semen entre porta y cubreobjeto, valorándose de acuerdo a la siguiente escala: 1: movimiento no perceptible, 2: movimiento débil con formación de alguna ola, 3 : movimiento lento con olas, 4: movimiento vigoroso con moderada rapidez de olas y remolinos, 5: denso, y movimiento muy rápido con olas y remolinos ${ }^{26}$.

Concentración espermática. El número de espermatozoides fue determinado en el semen fresco con una cámara de Neubauer ${ }^{6}$.

Lavado de semen. Las muestras de semen fueron centrifugadas a $300 \mathrm{X}$ g por $15 \mathrm{~min}$ para remover el plasma seminal, para lo cual se utilizó un medio de lavado a $37^{\circ} \mathrm{C}$, en una proporción de 1:10. El medio de lavado contenía los siguientes ingredientes: $1,60 \mathrm{~g}$ de TRIS (hidroximetil aminometano), 1,89 g de glucosa y $1,48 \mathrm{~g}$ de ácido cítrico en $100 \mathrm{ml}$ de agua destilada. Se separó el plasma seminal y el precipitado celular se resuspendió en un medio de dilución definitivo, cuyos componentes fueron los mismos del medio de lavado más $20 \%$ de yema de huevo y $6 \%$ de glicerol.

Adición de antioxidantes. Para la adición de antioxidantes en cada tratamiento se tomaron $20 \mathrm{ml}$ del medio de dilución definitivo formando cuatro grupos: grupo testigo, grupo con $5 \mathrm{mg} / \mathrm{ml}$ de vitamina $\mathrm{C}$, grupo con $5 \mathrm{mg} / \mathrm{ml}$ de vitamina E y grupo con $5 \mathrm{mg} / \mathrm{ml} \mathrm{de}$ cada una de las vitaminas $\mathrm{C}$ y E.

Criopreservación espermática. El semen diluido fue envasado en pajillas de $0,5 \mathrm{ml}$ y fueron selladas con perlas de cristal. La carga por pajilla fue de 100 millones de espermatozoides. Las pajillas fueron previamente rotuladas con el número de semental y el tratamiento, tras lo cual fueron puestas en bañomaría a $37^{\circ} \mathrm{C}$ y enfriadas a $5^{\circ} \mathrm{C}$ en un tiempo aproximado de 2,5 horas. Una vez alcanzados $\operatorname{los} 5^{\circ} \mathrm{C}$, las pajillas fueron colocadas en posición horizontal y expuestas a vapores de nitrógeno líquido durante 20 minutos a una altura aproximada de $5 \mathrm{~cm}$ por encima del nivel de nitrógeno. Inmediatamente después fueron sumergidas en el termo de nitrógeno líquido y almacenadas hasta su determinación ${ }^{7,14}$.

Descongelación de las muestras. La descongelación de las pajillas se realizó en bañomaría a $37^{\circ} \mathrm{C}$ durante 15 segundos.

Análisis de la condensación de la cromatina espermática. Para ello se utilizó el kit (Cycle Test ${ }^{\mathrm{TM}}$ Plus DNA Reagent kit, Cat. N ${ }^{\circ} 340242$, Becton Dickinson, San José, CA, USA), constituido por tres soluciones. La solución A contenía tripsina para la degradación enzimática y digestión de membranas y citoesqueleto celular; la solución B: un inhibidor de tripsina y ribonucleasa para degradar el ARN; y la solución C: yoduro de propidio (IP).

Preparación de las muestras. Las muestras de semen fueron analizadas antes y después de la congelación. Para su análisis se tomaron $10 \mu \mathrm{l}$ de semen de cada tratamiento, para obtener una concentración final de 2 millones de células espermáticas. Cada una de las muestras fue colocada en tubos de plástico y se les adicionó $2 \mathrm{ml}$ de amortiguador fosfato de sodio (PBS), se mezclaron en vórtex y posteriormente se centrifugaron a $300 \mathrm{X}$ g por 5 min y se retiró el sobrenadante, nuevamente se les adicionó $2 \mathrm{ml}$ de la misma solución amortiguadora, las muestras fueron centrifugadas a $400 \mathrm{Xg}$ por 5 minutos y el sobrenadante fue desechado; inmediatamente después se añadieron $250 \mu \mathrm{l}$ de la solución A del kit cycle test ${ }^{\mathrm{TM}}$ plus DNA reagent; se mezclaron suavemente y se dejaron incubar $10 \mathrm{~min}$ a temperatura ambiente. Transcurrido este tiempo se añadieron $200 \mu \mathrm{lde}$ la solución $\mathrm{B}$, se mezclaron suavemente y se incubaron por 10 minutos a temperatura ambiente y finalmente se agregó la solución $\mathrm{C}$ dejando incubar por otros $10 \mathrm{minu}-$ tos en la oscuridad y en refrigeración a $4^{\circ} \mathrm{C}$. Al cabo de este tiempo se analizaron por citometría de flujo.

Evaluación de estrés oxidativo. Para determinar el grado de estrés oxidativo, se evaluó la presencia de malondialdehído mediante la reacción del ácido tiobarbitúrico (TBA), antes y después de congelar las muestras. De cada tratamiento se tomaron $200 \mu \mathrm{l}$ y fueron diluidos con $600 \mu \mathrm{l}$ de agua destilada, (dilución 1:4) se mezclaron en vórtex y se centrifugaron a $2500 \mathrm{rpm}$, posteriormente se separó el sobrenadante y el paquete celular fue desechado. Se colocaron $40 \mu \mathrm{l}$ del sobrenadante de cada tratamiento en tubos de ensayo de cristal con tapa de rosca, junto con $60 \mu 1$ de agua bidestilada y $1 \mathrm{ml}$ de TBA al $0,375 \%$ en ácido clorhídrico (HCI) 0,2 $\mathrm{N}$. A continuación las muestras se hirvieron en bañomaría durante $15 \mathrm{~min}$, se dejaron enfriar y se les adicionaron $500 \mu \mathrm{l}$ de ácido clorhídrico $0,2 \mathrm{~N}$ y finalmente las muestras se leyeron en un espectrofotómetro a una longitud de onda $532 \mathrm{~nm}$. Cada tratamiento se realizó por duplicado, utilizándose 1,1,3,3 tetrametoxipropano como estándar. Los resultados fueron expresados en nanomoles de malondialdehído (MDA) por miligramo de proteína (nm MDA/mg de proteína) ${ }^{21}$.

Análisis estadístico. Las estadísticas descriptivas incluyeron media aritmética y desvío estándar $(\bar{x} \pm \mathrm{DE})$. La significación del grado de oxidación de los lípidos de la membrana plasmática y el grado de condensación de la cromatina espermática, antes y después de descongelar, fueron obtenidos por análisis de varianza (ANOVA) a una vía.

\section{RESULTADOS}

En la Tabla 1 se presentan los resultados de la motilidad espermática, después de la descongelación en todos los grupos analizados. La Tabla 2 muestra los resultados de la condensación-descondensación de la 
cromatina espermática, en los diferentes tratamientos; antes de congelar y después de descongelar. El ANOVA del grado de condensación de la cromatina antes y después del congelado reveló que las diferencias entre tratamientos fueron no significativas ( $p>0,05)$.

No hubo diferencias estadísticamente significativas entre los grupos analizados antes de congelar para la disminución del grado oxidativo $(\mathrm{p}>0,05)$, lo cual indica que la adición de antioxidantes no ejerció efecto protector en los ácidos grasos de las membranas plasmáticas de los espermatozoides.

Después de descongelar, el grupo testigo tuvo una protección 6,76 \% mayor que el grupo con vitamina $\mathrm{C}$, lo cual indica que la vitamina $\mathrm{C}$ sola tuvo un efecto pro-oxidante. El grupo con vitamina E reveló una protección $9,8 \%$ mayor al grupo testigo y $15,84 \%$ más alta que el grupo con vitamina $\mathrm{C}$, pero el efecto protector de la vitamina $\mathrm{E}$ fue menor que el proporcionado por la combinación de vitaminas $\mathrm{C}+\mathrm{E}$ que fue de un $25,5 \%$ mayor que el grupo testigo y $30,5 \%$ mayor que el grupo con vitamina $C$, así como una protección $17,44 \%$ mayor con la combinación de vitaminas $\mathrm{C}+\mathrm{E}$ comparado con el efecto de la vitamina $\mathrm{E}$ sola $(\mathrm{p}<0,05)$. En concordancia, la cantidad de malondialdehido (indicador de lipoperoxidación), expresada en nanomoles por miligramo de proteína, fue significativamente menor para la combinación de vitaminas $\mathrm{C}+\mathrm{E}$.

\section{DISCUSIÓN}

Se han adicionado antioxidantes al medio de dilución de semen de los machos reproductores para minimizar los daños causados por las ROS y evitar la peroxidación. Los antioxidantes investigados fueron súperoxido dismutasa, catalasa, citocromo $\mathrm{C}$, glutation peroxidasa, butil hidroxitolueno, butil hidroxianisol, n-propil galato, mesilato de deferoxamina, vitamina $\mathrm{E}$, vitamina $\mathrm{C}$ y albúmina sérica bovina, probados en semen de carnero, pavo, equino ${ }^{4}$, búfalo ${ }^{25} \mathrm{y}$ bovino 9, 10, 15, 16

Por otra parte la citometría de flujo puede valorar múltiples características celulares de manera precisa y objetiva, como células espermáticas apoptóticas ${ }^{1}$, viabilidad espermática, función de las mitocondrias y estructura de la cromatina ${ }^{11}$; también se puede evaluar la condensación de la cromatina espermática y la estabilidad del núcleo ${ }^{7,14}$, aunque la estructura de la cromatina puede variar entre los individuos y entre los eyaculados ${ }^{11}$. después de descongelar $(\overline{\mathrm{x}} \pm \mathrm{DE})$
Tabla 1. Promedio de motilidad de los espermatozoides expresado en \% después de descongelar el semen.

\begin{tabular}{cccc}
\hline testigo & vitamina $C$ & vitamina $\mathrm{E}$ & vitaminas $\mathrm{C}+\mathrm{E}$ \\
\hline 40 & 30 & 60 & 60 \\
45 & 40 & 50 & 40 \\
45 & 30 & 60 & 70 \\
40 & 30 & 50 & 70 \\
\hline
\end{tabular}

Cada número corresponde al promedio de 8 eyaculados de cada macho y de cada tratamiento (384 pajillas analizadas en total).

Tabla 2. Grados de condensación de la cromatina espermática antes de congelar y

\begin{tabular}{cccccccc}
\hline Test.1 & Test.2 & Vit.C1 & Vit.C2 & Vit.E1 & Vit.E2 & C+E1 & C+E2 \\
\hline 282.25 & 295.07 & 265.78 & 251.39 & 297.95 & 241.25 & 280.81 & 257.11 \\
272.65 & 289.36 & 206.86 & 269.26 & 288.11 & 289.52 & 243.76 & 280.92 \\
282.25 & 295.37 & 265.78 & - & 297.95 & 257.38 & 292.06 & 269.01 \\
289.74 & 273.75 & 289.97 & - & 305.24 & 286.38 & 271.40 & 272.40 \\
265.45 & - & 270.75 & - & 279.18 & - & - & - \\
\hline 278.46 & 288.48 & 259.82 & 260.32 & 293.68 & 268.60 & 272.0 & 269.86 \\
\pm 9.47 & \pm 10.23 & \pm 31.24 & \pm 12.63 & \pm 10.14 & \pm 23.26 & \pm 20.63 & \pm 9.86 \\
\hline
\end{tabular}

Test.1: testigo antes de congelar, Test.2: testigo después de descongelar, Vit.C1: vitamina C antes de congelar, Vit.C2: vitamina C después de descongelar, Vit.E1: vitamina E antes de ongelar, Vit.E2: vitamina $\mathrm{E}$ después de descongelar, $\mathrm{C}+\mathrm{E} 1$ : vitaminas $\mathrm{C}+\mathrm{E}$ antes de congelar, $\mathrm{C}+\mathrm{E} 2$ : vitaminas $\mathrm{C}+\mathrm{E}$ después de descongelar

La calidad de la estructura de la cromatina es un factor importante en la fecundación. En este estudio se encontró que no hubo diferencia en la condensación de la cromatina espermática antes de congelar y después de descongelar, lo cual contrasta con reportes que afirman que el semen de porcino congelado y descongelado tuvo una menor absorción de yoduro de propidio indicando una compactación o mayor condensación de la cromatina, comparado con espermatozoides frescos ${ }^{14}$. La fertilidad de los machos reproductores puede relacionarse con los defectos de la cromatina provocados por un aumento en la susceptibilidad a la descondensación, aunque durante la espermatogénesis y el transporte por el epidídimo hay una condensación de la cromatina espermática. Sin embargo, en el momento de la fecundación es necesario una descondensación, ya que alteraciones en la estructura de la cromatina son asociadas con infertilidad. La fertilidad del semen congelado es menor que la del semen fresco, la congelación causa apoptosis de las células espermáticas, fragmentación y mayor condensación del DNA espermático ${ }^{1}$. Además, la estructura de la membrana espermática sufre cambios durante el periodo de equilibrio, así como en el proceso de congelación y descongelación, disminuyendo la proporción de espermatozoides vivos.

En nuestro estudio se utilizó vitamina $\mathrm{C}$ y vitamina E por separado y en combinación. Son antioxidantes que se encuentran tanto en el medio intra como extracelular; el $\alpha$-tocoferol se localiza en las membranas celulares. En este análisis con la mezcla de estos antioxidantes hubo un efecto significativo en el man- 
tenimiento de la motilidad comparada con el grupo testigo, lo cual contrasta con lo reportado por autores que encontraron que la combinación de antioxidantes no mejora significativamente el mantenimiento de esta característica espermática ${ }^{4}$. En este trabajo, la combinación de antioxidantes $\mathrm{C}+\mathrm{E}$, redujo significativamente el grado de estrés oxidativo en relación al grupo testigo, después de descongelar los espermatozoides.

La vitamina $\mathrm{C}$ expresa uno de los mecanismos de defensa contra las ROS ${ }^{19}$; además, puede prevenir el daño oxidativo en macromoléculas semejantes al ADN, lípidos y proteínas por una protección antioxidante en fluidos biológicos de animales y seres humanos, pero no influencia el porcentaje de motilidad progresiva de los espermatozoides en almacenamiento, por una reducción del $\mathrm{pH}$. En semen de pavo, la vitamina $\mathrm{C}$ no mejoró la motilidad, lo cual concuerda con lo encontrado en este estudio, ya que al adicionar la vitamina $\mathrm{C}$ sola no hubo una diferencia en la motilidad de los espermatozoides, cuando se compararon con el grupo testigo. La suplementación de vitamina $\mathrm{C}$ y E como antioxidantes en el agua de bebida en conejos mejoró la calidad de semen al reducir la producción de radicales libres ${ }^{29}$, ya que hay una estabilidad oxidativa del semen, relacionada con el consumo de $\alpha$-tocoferol y de vitamina $\mathrm{C}^{13}$. La vitamina $\mathrm{E}$ puede tener efectos adversos sobre la motilidad de los espermatozoides, ya que puede actuar como antioxidante o pro-oxidante al inhibir o facilitar la peroxidación, esta actividad prooxidante del $\alpha$-tocoferol es prevenida por el ascorbato que actúa como un pro-antioxidante ${ }^{12}$.

En conclusión, el proceso de congelación-descongelación de semen, genera especies reactivas del oxígeno que dañan las membranas de los espermatozoides. En este estudio, la combinación antioxidante de vitaminas $\mathrm{C}+\mathrm{E}$ redujo el grado de oxidación de la membrana espermática de manera sinérgica, dando como resultado una mayor calidad en la conservación de los espermatozoides en estado congelado, con mantenimiento de la motilidad. Sin embargo, los antioxidantes utilizados no tuvieron efecto sobre la condensacióndescondensación de la cromatina espermática. Los resultados de este ensayo aportan a la optimización de la preservación del semen congelado de macho cabrío.

Agradecimiento. Al proyecto FOMES-98-35-28 de la UAM-Iztapalapa por su colaboración en el análisis por citometría de flujo.

\section{REFERENCIAS}

1. Anzar M, Liwei H, Buhr M, Kroetsch T, Pauls KP. 2002. Sperm apoptosis in fresh and cryopreserved bull semen detected by flow cytometry and its relationship with fertility. Biol Reprod 66: 354-360.

2. Azzi A, Breyer I, Feher M, Pastori M, Ricciarelli R, Spycher S, Staffieri M, Stocker A, Zimmer S, Zingg J. 2000. Specific cellular responses to alpha-tocopherol. $J$ Nutr 130: 1649-1652.
3. Ball BA, Vo AT, Baumber J. 2001. Generation of reactive oxygen species by equine spermatozoa. Am J Vet Res 62 : 508-515.

4. Ball BA, Medina V, Gravance CG, Baumber J. 2001. Effect of antioxidants on preservation of motility, viability and acrosomal integrity of equine spermatozoa during storage at $5^{\circ} \mathrm{C}$. Theriogenology 56: 577-589.

5. Ball BA, Baumber J, Sabeur K. 2002. Role of reactive oxygen species on normal and abnormal function of equine spermatozoa. Theriogenology 58: 299-300.

6. Barrios B, Pérez R, Gallego M, Tato A, Osada J, Muiño-Blanco T, Cebrián-Pérez JA. 2000. Seminal plasma proteins revert the cold-shock damage on ram sperm membrane. Biol Reprod 63: 1531-1537.

7. Bag SJ, Mathur AK, Rawat PS, Mittal JP. 1999. Effect of thawing temperature on motion characteristics of frozen-Thawed ram spermatozoa. Indian J Anim Sci 69: 16-18.

8. Baumber J, Ball BA, Linfor JJ, Meyers SA. 2002. Reactive oxygen species and cryopreservation promote deoxyribonucleic acid (DNA) damage in equine sperm. Theriogenology 58: 301-302.

9. Bilodeau JF, Blanchette S, Gagnon C, Sirard MA. 2001. Thiols prevent $\mathrm{H}_{2} \mathrm{O}_{2}$ mediated loss of sperm motility in cryopreserved bull semen. Theriogenology 56: 275-286.

10. Bilodeau JF, Blanchette S, Cormier N, Sirard MA. 2002. Reactive oxygen species-mediated loss of bovine sperm motility in yolk tris extender: protection by pyruvate, metal chelators and bovine liver or oviductal fluid catalase. Theriogenology 57: 1105-1122.

11. Bochenek M, Smorag Z, Pilch J. 2001. Sperm chromatin structure assay of bulls qualified for artificial insemination. Theriogenology 56:557-567.

12. Carr AC, Zhu BZ, Frei B. 2000. Potential antiatherogenic mechanisms of ascorbate (vitamin C) and alpha- tocopherol (vitamin E). Circ Res 87: 349-354.

13. Castellini C, Lattaioli P, Bernardini M, Dal Bosco A. 2000. Effect of dietary $\alpha$-tocopheryl acetate and ascorbic acid on rabbit semen stored at $5^{\circ} \mathrm{C}$. Theriogenology 54 : 523-533.

14. Córdova A, Pérez-Gutiérrez JF, Lleó B, García-Artiga C, Alvarez A, Drobchak V, Martín-Rillo S. 2002. In vitro fertilizing capacity and chromatin condensation of deep frozen boar semen packaged in 0.5 and $5 \mathrm{ml}$ straws. Theriogenology 57: 2119-2128.

15. Foote RH, Brockett CC, Kaproth MT. 2002. Motility and fertility of bull sperm in whole milk extender containing antioxidants. Anim Rep Sci 71: 13-23.

16. Frei B. 1999. Molecular and biological mechanisms of antioxidant action. Faseb $J$ 13: 963-964.

17. Hicks JJ. 2001. Bioquímica, McGraw-Hill, México, 900 p.

18. Holt WV. 2000. Basic aspects of frozen storage of semen. Anim Reprod Sci 62: 3-22.

19. Kankofer M. 2001. Non-enzymatic antioxidative defense mechanisms again reactive oxygen species in bovine-retained and not-retained placenta: vitamin $\mathrm{C}$ and glutathione. Reprod Dom Anim 36: 203-206. 
20. Leboeuf B, Restall B, Salamon S. 2000. Production and storage of goat semen for artificial insemination. Anim Reprod Sci 62: 113-141.

21. Lowry OH, Rosebrough NJ, Farr AL, Randall RJ. 1951. Protein measurement with the folin phenol reagent. $J$ Biol Chem 193: 265-275.

22. Lysiak JJ, Nguyen QA, Turner TT. 2002. Peptide and nonpeptide reactive oxygen scavengers provide partial rescue of the testis after torsion. $J$ Androl 23: 400-409.

23. Ollero M, Gil-Guzman E, Lopez MC, Sharma RK, Agarwal A, Larson K, Evenson D, Thomas JA, Alvarez CJ. 2001. Characterization of subsets of human spermatozoa at different stages of maturation: implications in the diagnosis and treatment of male infertility. Hum Reprod 16: 1912-1921.

24. Salamon S, Maxwell WM. 2000. Storage of ram semen. Anim Reprod Sci 62: 77-111.

25. Sansone G, Nastri MJ, Fabbrocini A. 2000. Storage of buffalo (Bubalus bubalis) semen. Anim Reprod Sci 62: 5576.
26. Shamsuddin M, Amiri Y, Bhuiyan MM. 2000. Characteristics of buck semen with regard to ejaculate numbers, collection intervals, diluents and preservation periods. Reprod Dom Anim 35: 53-57

27. Sommer D, Fakata KL, Swanson SA, Stemmer PM. 2000. Modulation of the phosphatase activity of calcineurin by oxidants and antioxidants in vitro. Eur J Biochem 267: 2312-2322.

28. Wang XL, Rainwater DL, Vandeberg JF, Mitchell BD, Mahaney MC. 2001. Genetic contributions to plasma total antioxidant activity. Arterioscler Thromb Vasc Biol 21: 1190-1195.

29. Yousef MI, Abdallah GA, Kamel KI. 2003. Effect of ascorbic acid and vitamin E supplementation on semen quality and biochemical parameters of male rabbits. Anim Repr Sci 76: 99-111.

30. Yves C. 2000. Free radicals are in fact potent oxidative stress and Alzheimer disease. Am J Clin Nutr 71: 621S629 S. 